\title{
Andalucía: identidad y cultura. Recuperación, conocimiento, concienciación
}

Juan Antonio Lacomba

Catedrático de Historia Económica de la E.U. de Ciencias Empresariales de la Universidad de Málaga

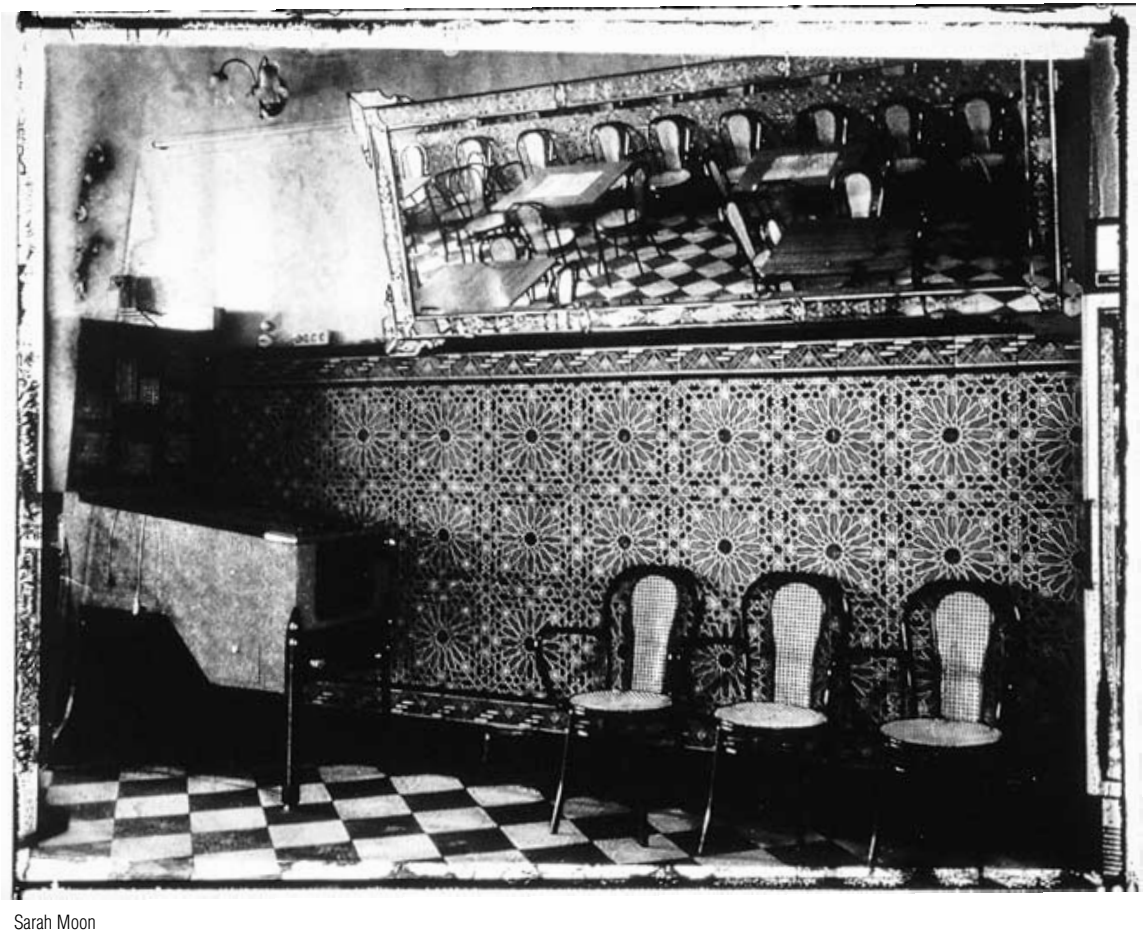

La reflexión sobre la posible Andalucía del siglo XXI, desde el enfoque de la cultura, implica, mínimamente, el abordar dos niveles: uno primero, de manera más general, sobre la identidad y la cultura del pueblo andaluz, en tanto que resultantes históricos, y la necesidad de su recuperación, conocimiento y asunción plenas en el marco de la globalización; otro segundo, de forma más precisa, sobre qué hacer en este campo para que, además de su fundamental valor social como sustentador de la conciencia de pueblo, pueda ser un activo importante que ayude al desarrollo de Andalucía en el nuevo siglo. En estas dos líneas apuntadas se insertan las reflexiones que a continuación se exponen.

\section{Andalucía: identidad y cultura. Ocultación, desvirtuamiento y recuperación}

Existen en España distintas realidades regionales, con desiguales niveles de desarrollo, que determi- nan tendencias diferentes a largo plazo. Ello supone en algunas regiones, y es el caso de Andalucía, la necesidad de políticas económicas públicas destinadas a remover los obstáculos que impiden o dificultan que una región avance más rápidamente hacia la aminoración de su nivel de atraso relativo (políticas de convergencia).

Dichos "obstáculos" pueden ser de diverso tipo. Entre ellos hay cuatro más significativos: los relacionados con la estructura productiva; los vinculados a carencias de infraestructura; los debidos a insuficiencia de capital humano; los referentes a cuestiones sociales. Aunque en buena medida todos los señalados son aplicables a Andalucía, cabe aceptar que el tema de la identidad y la cultura, y de sus problemas, que aquí nos interesa, se puede situar en el último grupo apuntado. En consecuencia, ha de haber en Andalucía una política pública que encare sin titubeos un programa de recuperación, conocimiento, concienciación y proyección de esta fundamental dimensión 
En suma, hay que plantear una política pública que valorice adecuadamente las cuestiones de identidad, cultura y multiculturalidad andaluza como un activo decisivo: de un lado, en su dimensión colectiva social, para la recuperación de Andalucía como pueblo; de otro, en su posible vertiente económica, para ayudar a su más pronta y real convergencia con las áreas más desarrolladas, ya que ambos objetivos, en el caso andaluz, pueden encontrarse hoy estrechamente relacionados.

de la realidad andaluza, que sea además capaz de movilizar, en esta misma dirección, al sector privado.

La identidad y la cultura andaluza, en definitiva, su etnicidad, es resultado del proceso histórico de Andalucía. En este sentido, se deben significar algunos "marcadores de identidad" y elementos sustentadores de su formación cultural: a) la secular persistencia de una economía de base agraria, por lo que "la cuestión de la tierra" (y "la lucha por la tierra") constituye un referente simbólico del pueblo andaluz; b) el consecuente predominio de unas relaciones sociales de producción caracterizadas por la permanencia de la dominación de una minoría (históricamente, la minoría propietaria) sobre una mayoría explotada; c) la existencia histórica de una multiculturalidad, resultado de la presencia en Andalucía de diferentes etnias, asumida, integrada y transformada en componente estructural de la propia cultura andaluza, lo que explica que la convivencia con otros pueblos y la solidaridad social sean aspectos sustanciales de la identidad andaluza. una periferia del centro. En lo social, la clase dominante andaluza, la oligarquía agraria, clase "desregionalizada", se alía con los grupos de poder nacionales para controlar, en beneficio de sus propios intereses, el aparato del Estado. En lo cultural, se produce la alienación progresiva del pueblo andaluz. En su fase final, la cultura andaluza se enajena del pueblo andaluz, se le "expropia", para ser "sobredimensionada" como cultura española. Mediante este proceso se enmascara y falsea la realidad de Andalucía: se difumina su identidad, se desvirtúa su cultura y se elimina su conciencia de pueblo.

Ante este despliegue histórico sumariamente esbozado, cuyas consecuencias siguen vigentes en buena medida en la Andalucía de hoy, es precisa, de cara al nuevo siglo, una "reapropiación" por el pueblo andaluz de su cultura, para así "reencontrar" y asumir su identidad colectiva, valor imprescindible para enfrentarse al futuro "globalizado" en construcción. Desde estos supuestos, es necesaria la recuperación por Andalucía de la memoria y la conciencia

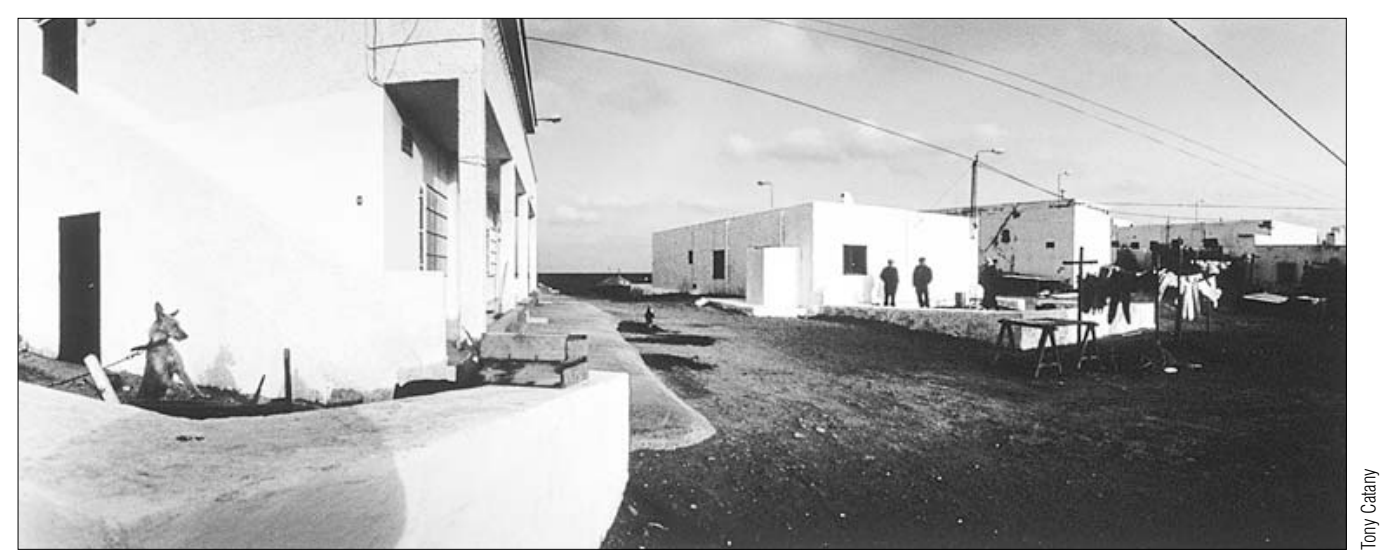

Con el despliegue del capitalismo se producirá la dominación económica de Andalucía, que dará lugar a su subordinación social, lo que conducirá a su alienación cultural. En lo económico, el proceso de dominación implicará fundamentalmente la periferización de Andalucía, tanto a escala nacional, como internacional: Andalucía se convertirá en periferia de de pueblo, mediante la plena reconstrucción y conocimiento de su historia y de su cultura, fundamentos de su etnicidad. Una política en este sentido se debe entender no sólo como una línea de intervención cultural, sino como un medio, junto con otros, cuyo fin es superar la situación de dependencia de Andalucía. 
En suma, hay que plantear una política pública que valorice adecuadamente las cuestiones de identidad, cultura y multiculturalidad andaluza como un activo decisivo: de un lado, en su dimensión colectiva social, para la recuperación de Andalucía como pueblo; de otro, en su posible vertiente económica, para ayudar a su más pronta y real convergencia con las áreas más desarrolladas, ya que ambos objetivos, en el caso andaluz, pueden encontrarse hoy estrechamente relacionados. Expresado de otra manera: en el proceso de globalización que se afianza, difícilmente se superará la dependencia y el atraso de Andalucía si no se recupera y afirma su etnicidad y su conciencia de pueblo, y se utilizan como instrumentos de impulso colectivo en esa dirección.

\section{Andalucía de cara al siglo XXI. Reflexiones sobre el papel de la cultura}

Como se ha señalado, para la Andalucía del siglo XXI debe de ser una cuestión prioritaria recuperar su etnicidad. Se trata de no quedar diluida ("desidentificada") en el proceso acelerado de globalización despersonalizadora, hecho que, además de otros, viene a propiciar la articulación subordinada de unos pueblos, regiones o espacios - las "áreas dominadas" - a otros pueblos o espacios - las "áreas dominantes" -. Ello implica: a) de un lado, recuperar la cultura propia, para conocerse, y transmitirla, reconstruyendo en los andaluces la conciencia de pueblo; b) de otro lado, y a partir de lo anterior, la proyección de Andalucía, hacia dentro y hacia fuera, cimentada en la recuperación de su pasado y en la plena asunción de su identidad, como un valor social y un activo económico.



Con todo ello se trata de encontrarse con el presente, desde el restablecimiento del pasado, para plantear la proyección en el futuro. Porque realmente ésta es la cuestión: qué hacer con un activo fundamental de Andalucía -su realidad como pueblo; su cultura y patrimonio cultural como expresión del mismo -en el nuevo siglo que se avecina. En este sentido, como iniciales propuestas sumarias, a desarrollar y precisar, se pueden apuntar las siguientes:
I. Recuperar, estudiar y proyectar, hacia dentro y hacia fuera de Andalucía, su amplio y rico patrimonio cultural, que es expresión de la multiculturalidad histórica andaluza, y pone de manifiesto el fenómeno de la convivencia de etnias diferentes en su territorio;

2. Propiciar por este medio a los andaluces el conocerse y concienciarse como pueblo, asumiendo su identidad como un componente necesario para su pleno desarrollo en un mundo en proceso de globalización y de despersonalización;

3. Plantear toda esta riqueza como un activo, no sólo cultural, sino también económico, capaz de desplegar en torno a él todo un conjunto de acciones que propicien, desde este flanco, la recuperación general de Andalucía.

Desde estos supuestos de base, se hacen las siguientes consideraciones:

\section{Integración desde la propia afirmación.}

Andalucía es una "construcción histórica", cuyo presente es el resultado del proceso. Se trata, en el nuevo siglo, de integrarse en un mundo en constante cambio, sin diluirse en él. Es, pues, necesario reencontrar los activos que hagan posible el desarrollo pleno de Andalucía en ese próximo futuro, a partir de unos "marcadores" de su identidad específica, que propicien su afianzamiento como pueblo y el mejor despliegue de sus potencialidades como tal pueblo.

\section{Economía de la cultura.}

La identidad, a más de factor definidor de pueblo, es un fundamental activo social. En este sentido, la cultura propia es un bien susceptible de promover un desarrollo económico articulado en torno a ella. La cultura es, pues, capaz de generar una actividad que, al tiempo que la defiende y potencia, propicia todo un conjunto de servicios a ella vinculados. En consecuencia, la defensa y promoción del patrimonio en Andalucía es una política que, además de recuperar identificadores culturales, puede impulsar una economía que ayude al desarrollo de áreas y espacios.

\section{Cultura y desarrollo.}

Se trata, por todo ello, no sólo de estudiar y conocer la propia cultura y la propia identidad, como señales de la realidad andaluza en el tiempo, sino también de articular una estrategia para la conservación y difusión del patrimonio, desde la que proponer actividades que impulsen el crecimiento económico de Andalucía. De esta manera, los avances en el conocimiento de la cultura andaluza y en la recuperación del patrimonio no significarán la recuperación de un bien "ocioso", sino que serán realmente la potenciación de un activo que permite a los andaluces tomar conciencia de su identidad y posibilita una determinada economía. Así, mediante una adecuada política pública, se aúnan cultura y desarrollo.

\section{Política cultural/patrimonial para Andalucía.}

En este sentido, y desde la óptica planteada, ¿qué hacer en la Andalucía del siglo XXI?. El presupuesto de partida es entender el gasto en cultura, a más de 


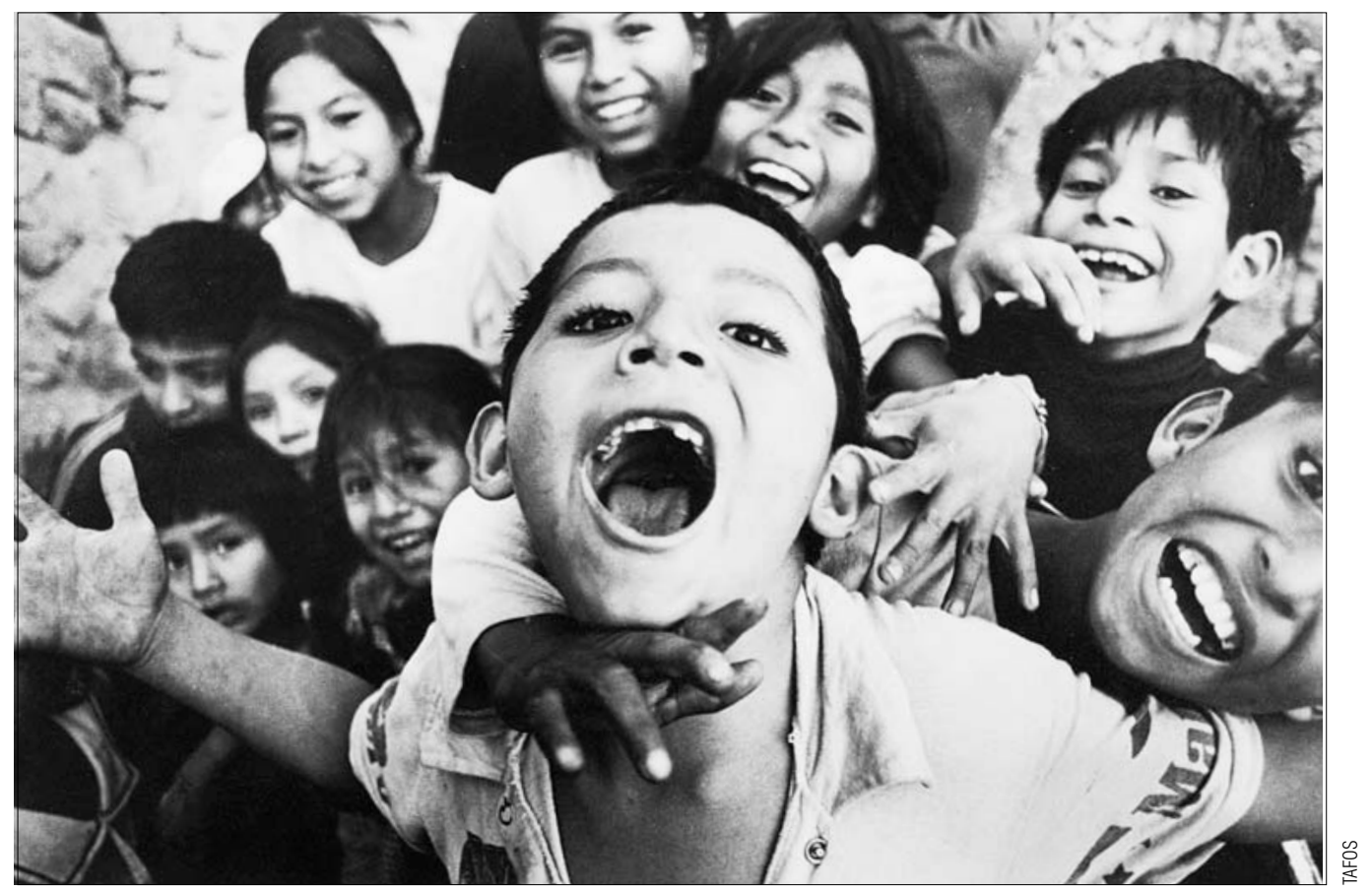

como un obligatorio servicio a la sociedad, como una inversión productiva a medio y largo plazo. Según este supuesto, el gasto en cultura debe tener como eje de articulación la recuperación de la conciencia de identidad andaluza; y el gasto en patrimonio debe considerarse una inversión social necesaria que, además, permite la utilización de los bienes patrimoniales como medios de generar una actividad económica.

A partir de la fundamentación expuesta, ¿qué objetivos básicos se proponen?: I) Recuperar la historia y la cultura de Andalucía; 2) Propiciar el conocimiento de los andaluces de su identidad cultural; 3) Buscar la inserción de la política cultural y patrimonial en el modelo de crecimiento de Andalucía. Para alcanzar estos objetivos, se postula una política pública autonómica articulada en los siguientes núcleos:

A. Estudio sistemático y reglado en los niveles educativos de la historia y la cultura de Andalucía:

a) Nivel no universitario: introducción de una disciplina de Historia y cultura de Andalucía

b) Nivel universitario: creación de una especialización universitaria de estudios sobre Andalucía

B. Apoyo prioritario a las investigaciones de diverso tipo orientadas al mejor conocimiento de Andalucía:

a) Creación de un "Instituto de Cultura Andaluza" que, con diferentes Secciones, potencie estas investigaciones, conceda ayudas para la formación de especialistas y coordine trabajos en este sentido

b) Apoyo a las líneas de investigación sobre Andalucía de los Departamentos Universitarios andaluces

C. Impulso al conocimiento y difusión de la cultura andaluza mediante la configuración de un "Sistema de Bibliotecas, Archivos y Museos" de Andalucía.

Esta política autonómica debe ser complementada: de un lado, por una política local que apoye con re- cursos la recuperación de su propio patrimonio cultural, colabore en su sostenimiento y propicie su estudio y difusión; de otro lado, por el sector privado, creando los mecanismos que hagan posible su colaboración en la recuperación, restauración y mantenimiento del patrimonio cultural, su ayuda para su estudio y conocimiento y su patrocinio en publicaciones que favorezcan su amplia difusión.

\section{Consideraciones finales}

Volvemos al principio: de cara al nuevo siglo es necesaria una política pública regional en la que el sector cultural no sea simplemente "decorativo", sino que esté entendido como un activo social y económico importante para la recuperación general de Andalucía. Sus tres fundamentos pueden ser:

I. La concepción de Andalucía como pueblo en el contexto de la plural realidad que es España. En este sentido, la idea clave sería conocer y asumir quien se ha sido y se es, para proponer un horizonte de futuro;

2. La multiculturalidad como realidad y estructura de fondo de la cultura andaluza. Desde esta perspectiva, la cuestión sería: la cultura andaluza es resultado de la convivencia de culturas, lo que implica la existencia histórica de Andalucía como mundo abierto y tolerante;

3. La articulación de una economía de la cultura que, a la vez que permite su recuperación y conocimiento, la entiende como un recurso en favor del desarrollo de Andalucía. Este enfoque significa: la cultura debe considerarse un servicio a la sociedad andaluza, pero también un sector susceptible de un tratamiento económico que ayude al crecimiento de Andalucía. 